\title{
Análisis de los hábitos de estudio en estudiantes de nivel superior, un caso de estudio
}

\section{Analysis of study habits in higher level students, a case study}

MIRELES-MEDINA, Antonia†*, ARREDONDO-SALCEDO, Daniel, CASTAÑEDA-DELGADO, Jaime y MOTA-GARCÍA, María Juana

Instituto Tecnológico Superior Zacatecas Norte, México.

ID $1^{\text {er }}$ Autor: Antonia, Mireles-Medina / ORC ID: 0000-0001-9773-9108, CVU CONACYT ID: 299436

ID $1^{\text {er }}$ Coautor: Daniel, Arredondo-Salcedo / ORC ID: 0000-0003-3236-4880, CVU CONACYT ID: 316030

ID $2^{\text {do }}$ Coautor: Jaime, Castañeda-Delgado / ORC ID: 0000-0001-9786-4128, CVU CONACYT ID: 252889

ID $3^{\text {er }}$ Coautor: María Juana, Mota-García / ORC ID: 0000-0003-1127-1116, CVU CONACYT ID: 636080

DOI: $10.35429 / J H S .2020 .11 .4 .1 .10$

Recibido Enero 10, 2020; Aceptado Junio 30, 2020

\begin{abstract}
Resumen
Es responsabilidad de las Instituciones de Educación Superior ofertar educación de calidad a sus estudiantes de modo que con ello se logre que sean más competitivos en el área específica donde han decido formarse, para tal efecto es importante que los docentes y los tutores académicos tengan conocimiento sobre los hábitos de estudio que poseen sus alumnos, así como también conocimiento de la existencia de objetos de aprendizaje electrónicos genéricos que apoyen a mejorar sus experiencias o hábitos de estudio en caso de ser necesario, esto con la finalidad de que se genere dentro del aula estrategias que impulsen y motiven a los estudiantes para que decidan comprometerse con su estudio y obtengan un rendimiento académico aceptable. Ya que, las técnicas o formas de estudio que poseen están directamente relacionadas con su rendimiento. Evitando de esta forma la reprobación en las asignaturas que semestre con semestre van cursando y el incremento en la deserción escolar. El objetivo de la presente investigación es efectuar un análisis de los hábitos de estudio en 17 estudiantes de Ingeniería en Sistemas Computacionales para identificar áreas de oportunidad y de esta manera implementar costumbres y estrategias idóneas en ellos para la disminución de las malas prácticas en su estudio.
\end{abstract}

Hábitos de estudio, rendimiento académico, objetos de aprendizaje electrónicos

\begin{abstract}
It is the responsibility of Higher Education Institutions to offer quality education to their students in such a way as to make them more competitive in the specific area where they have decided to train, for this purpose it is important that teachers and academic tutors have knowledge about the study habits that their students have, as well as knowledge of the existence of generic electronic learning objects that help to improve their experiences or study habits if necessary, this in order to generate strategies within the classroom that encourage and motivate students to decide to commit to their study and obtain acceptable academic performance. Since, the techniques or forms of study they possess are directly related to their performance. Avoiding in this way the failure in the subjects that semester with semester are taking and even the decrease in school dropout. The objective of this research is to carry out an analysis of study habits in 17 Computer Systems Engineering students to identify areas of opportunity and in this way to implement suitable customs and strategies in them to reduce bad practices in their study.
\end{abstract}

Study habits, Academic performance, Electronic learning objects

Citación: MIRELES-MEDINA, Antonia, ARREDONDO-SALCEDO, Daniel, CASTAÑEDA-DELGADO, Jaime y MOTA-GARCÍA, María Juana. Análisis de los hábitos de estudio en estudiantes de nivel superior, un caso de estudio. Revista de Educación Superior. 2020. 4-11:1-10.

*Correspondencia al Autor (Correo Electrónico: mirelesmed_7@ @hotmail.com)

$\dagger$ Investigador contribuyendo como primer autor. 


\section{Introducción}

En términos generales, el hábito es el conjunto de costumbres, formas y maneras de percibir, sentir, juzgar, actuar y pensar de una persona (Perrenod, 1996). Según el Diccionario de la Real Academia Española (2020), un hábito es "un modo especial de proceder o conducirse adquirido por repetición de actos iguales o semejantes, $\mathrm{u}$ originado por tendencias instintivas". Los autores de la presente investigación conceptualizan el hábito como una práctica constante que una persona realiza en su vida cotidiana.

En términos específicos los hábitos de estudio (HE) son los métodos y estrategias que acostumbra a usar el estudiante para asimilar conocimientos, su aptitud para evitar distracciones, su atención al material específico y los esfuerzos que realiza a lo largo de todo el proceso (Cartagena, 2008). Así mismo, "el hábito de estudio son modos constantes de actuación con que el estudiante reacciona ante los nuevos contenidos, para conocerlos, comprenderlos y aplicarlos. Podemos enumerar, como los más importantes los siguientes: aprovechar el tiempo de estudio, lograr condiciones idóneas, desechar los elementos perturbadores, plantear eficazmente el trabajo, seleccionar correctamente las fuentes de información y documentación, presentar adecuadamente los resultados, dominar las técnicas de observación, atención, concentración y relajación” (Sánchez, 2002).

Además, otros autores consideran que entre los hábitos de estudio que mejoran el desempeño académico de los estudiantes en educación superior destacan los siguientes: la administración del tiempo; las habilidades cognitivas como la memoria, la atención y la concentración; la comprensión de lectura; los apuntes de clase; la redacción; el concepto de sí mismo; la motivación y voluntad; las relaciones interpersonales y el trabajo en equipo (Torres $e t$ al., 2009).

Autores como Bajwa, Gujjar, Shaheen y Ramzan (2011) mencionan que un estudiante no puede usar habilidades de estudio eficaces, hasta que no tenga buenos hábitos y argumentan que un individuo aprende con mayor rapidez y profundidad que otros debido a sus acertados hábitos de estudio.
Al respecto los autores del presente artículo consideran que es de suma importancia comprender que los estudiantes emergen de diferentes contextos o entornos y como tal también debe entenderse que cada uno ellos se han desarrollado en culturas propias de su lugar de procedencia y de las instituciones que han egresado, sin duda alguna existe una gran diversidad de contextos que influyen en la adquisición de los hábitos de estudio.

El interés por los hábitos de estudio de los alumnos universitarios se ha convertido en un tema de actualidad tanto a nivel nacional como internacional debido al alto porcentaje de reprobación (Villegas, Muñoz, \& Villegas, 2016). Situación de la que no es ajena el Tecnológico Nacional de México, Campus Zacatecas Norte de donde emana este estudio, debido a que regularmente en los cuatro primeros semestres se presentan índices de reprobación que se encuentran por encima de la media nacional, situación que en ocasiones se agudiza y viene a impactar en otro indicador que es la deserción escolar.

Actualmente, los hábitos de estudio han sido objeto de investigación por varios autores, tal es el caso de la siguiente investigación "Comportamiento de los estudiantes en función a sus hábitos de estudio" realizada por Escalante, Escalante, Linzaga y Merlos (2008), donde se evaluaron los hábitos de estudio de los estudiantes de la carrera de Ingeniero Agrónomo Zootecnista del Centro de Estudios Profesionales del Colegio Superior Agropecuario del Estado de Guerrero, México. El cual obtuvieron los valores de los hábitos de estudio del promedio de la suma de cuatro variables: estrategias de higiene, condición de materiales, estrategias de estudio y capacidad de estudio. Concluyeron que el aprendizaje del estudiante depende de su capacidad intelectual, de su estado emocional y de sus hábitos de estudio. Variables que consideramos elementales para lograr un buen rendimiento académico en los estudiantes.

En la investigación llevada a cabo con estudiantes universitarios de primer ingreso se determinó que la mayoría de los estudiantes analizados no usan apropiadas técnicas de estudio y no distribuyen su tiempo de forma adecuada. 
Dentro de los aspectos cognitivos que se relacionan con el bajo rendimiento académico identifican que los estudiantes de primer ingreso en el ámbito universitario en su mayoría no entienden los contenidos de asignaturas que utilizan números y las que utilizan la lógica (Contreras, Caballero, Palacio, \& Pérez, 2008). En el caso de los estudiantes de Ingeniería en Sistema Computacionales del Tecnológico Nacional de México, Campus Zacatecas Norte les pasa lo mismo en las asignaturas como: Cálculo Diferencial, Cálculo Integral, Cálculo Vectorial, Algebra Lineal, Ecuaciones Diferenciales, Fundamentos de Programación, Programación Orientada a Objetos y Estructura de Datos que son donde regularmente presentan dificultad para aprender.

Es importante mencionar que tales materias son cursadas en los primeros cuatro semestres de su carrera profesional y se ha presentado la situación de que estudiantes de octavo semestre, aún están cursando tales asignaturas en cursos de repetición o especial. Aunado a la complejidad de las asignaturas, los tutores académicos han identificado por medio de instrumentos de recolección de datos prediseñados que tienen deficiencias en los HE $y$ que hacen falta estrategias efectivas para fomentarlos, debido a que tal escenario contribuye a la reprobación y a la deserción.

Mientras que Hernández, Rodríguez y Vargas (2012) en su trabajo de investigación titulado "Los hábitos de estudio y motivación para el aprendizaje de los alumnos en tres carreras de ingeniería", realizado con 414 alumnos de un Tecnológico Federal de México, concluyeron que los estudiantes tienen problemas por la organización y planeación del estudio, las técnicas de memorización, la comprensión de lectura al estudiar en casa, y la carencia de estrategias para incrementar la motivación y autoestima.

Cabe destacar que la motivación que tiene el estudiante frente al estudio es de peculiar importancia, ya que sin ésta no habrá poder humano que impulse su perseverancia en sus estudios. Un estudiante desmotivado va a tender al fracaso escolar y esto es lo que se pretende evitar por medio de estrategias que impulsen el deseo de aprender y de adquirir conocimientos.
En la investigación conformada por 135 estudiantes de una universidad privada de Lima se midieron los hábitos de estudio con el Inventario de Hábitos de Estudio de Pozar y la autoeficacia con la Escala de Autoeficacia General de Schawzer, encontrando una relación significativa entre la autoeficacia percibida y los hábitos de estudio, siendo esta asociación mayor con las escalas de asimilación de contenidos y condiciones ambientales de estudio (Acevedo, Torrres y Tirado, 2015).

En otras investigaciones como la de Cruz Núñez y Quiñones Urquijo (2016) realizada en la Facultad de Enfermería Poza Rica-Tuxpan, con una población de 397 estudiantes inscritos en el periodo febreroagosto 2010. Utilizando el tipo de muestreo probabilístico estratificado con 79 estudiantes, aplicaron el inventario de hábitos de estudio de Gilbert Wrenn y el rendimiento académico considerado como el promedio que obtuvieron en el semestre anterior. Observaron una correlación significativa entre los hábitos de estudio con el rendimiento académico, por el cual proponen diseñar una estrategia metodológica para trabajar los hábitos de estudio desde el ingreso del estudiante a la universidad en aras de mejorar el rendimiento académico. Por tal razón los autores del presente estudio pretenden también indagar sobre la existencia de objetos de aprendizaje electrónicos genéricos que ayuden a mejorar los HE y como consecuencia mejorar el rendimiento académico.

Por último, en la Investigación realizada por Chilca (2017) la población estuvo conformada por 196 estudiantes de la Facultad de Ingeniería de la Universidad Tecnológica del Perú (UTP), matriculados en el curso de Matemática Básica I, durante el semestre académico 2016 - III y el tamaño de la muestra fue de 86 estudiantes. Se utilizó el inventario de autoestima (elaborado por Stanley Coopersmith), el Inventario de hábitos de estudio (elaborado por Luis Vicuña Peri) y el reporte de los promedios de las calificaciones de los estudiantes. Los resultados evidenciaron que no existe una influencia significativa entre la autoestima y el rendimiento académico, pero sí entre los hábitos de estudio y el rendimiento académico de los estudiantes, por lo que concluye que si los estudiantes mejoran sus técnicas de estudio entonces se incremente su rendimiento académico universitario. 


\section{Problema}

Actualmente, los docentes del Tecnológico Nacional de México, Campus Zacatecas Norte no diseñan estrategias didácticas idóneas o adecuadas para mejorar los hábitos de estudio en los estudiantes y como consecuencia mejorar su rendimiento académico, ya que desconocen cuál es su comportamiento en este rubro y sí en realidad los alumnos poseen e implementan formas o prácticas de estudio que contribuyan para facilitar el aprendizaje y puedan aprender en el aula y en su casa sin dificultad.

Regularmente, en los primeros cuatro semestres los tutores académicos son los encargados de identificar los hábitos de estudio y de entregar un informe específico a la Coordinación de Tutorías, al Departamento de Desarrollo Académico y a las Jefaturas de División correspondientes de cada una de las carreras que oferta el Campus en mención, sin embargo, tal informe no llega a manos de los docentes que les imparten las asignaturas y los departamentos que lo reciben tampoco impulsan estrategias para que se mejoren los HE en sus estudiantes. De aquí que surgen los siguientes cuestionamientos: ¿Cuáles son los hábitos de estudio que poseen los estudiantes de Ingeniería en Sistemas Computacionales del Campus Zacatecas Norte?, ¿existen objetos de aprendizaje electrónicos genéricos que ayuden a mejorar los HE? y ¿qué características deben reunir los objetos de aprendizaje electrónicos genéricos?

\section{Objetivo general}

Analizar los hábitos de estudio en 17 estudiantes de Ingeniería en Sistemas Computacionales del Tecnológico Nacional de México, Campus Zacatecas Norte para identificar áreas de oportunidad e implementar en ellos costumbres y estrategias didácticas adecuadas tales como objetos de aprendizaje electrónicos genéricos para la disminución de las malas prácticas en su estudio.

\section{Objetivos específicos}

Aplicar el cuestionario de hábitos de estudio de José Luis Díaz Vega a los estudiantes de Ingeniería en Sistemas Computacionales del Tecnológico Nacional de México, Campus Zacatecas Norte.
Identificar los hábitos de estudio de los estudiantes de Ingeniería en Sistemas Computacionales del Tecnológico Nacional de México, Campus Zacatecas Norte.

- Indagar sobre la existencia de objetos de aprendizaje electrónicos que apoyen al mejoramiento de los $\mathrm{HE}$ en los estudiantes de Ingeniería en Sistemas Computacionales del Tecnológico Nacional de México, Campus Zacatecas Norte.

Presentar un análisis de los resultados obtenidos en los estudiantes de Ingeniería en Sistemas Computacionales del Tecnológico Nacional de México, Campus Zacatecas Norte.

\section{Justificación}

Como lo afirman (Gutiérrez, Pacheco Amigo, \& Rodríguez García, 2017) "Los hábitos de estudio son el medio adecuado para conseguir las condiciones para una mejor educación", de tal forma que consideramos importante conocer sí los estudiantes poseen o no hábitos de estudio, debido a que primeramente tienen que identificarse para posteriormente implementar estrategias que apoyen a mejorar su forma de aprender y de adquirir conocimientos. De modo que su rendimiento académico no se vea afectado. Castejón (2014, p. 20), citando a Tourón, considera al rendimiento académico como: "Un resultado del aprendizaje producido por el estudiante, el producto de una suma de elementos, aún no del todo conocidos, que actúan sobre y desde la persona que aprende.

El rendimiento es, pues, un producto de factores que se sitúan dentro y fuera del individuo". De igual forma dice en su obra Aprendizaje y rendimiento académico que: "La concepción del rendimiento como producto educativo tiene su máxima expresión en la consideración de la función productiva que la educación tiene para un país, como se puso de manifiesto en la conferencia que, con el título Indicadores de la calidad de los sistemas educativos: una perspectiva internacional que se celebró en Roma en 1990 organizada por la Asociación Internacional para la Evaluación de los Logros Educativos, con sus siglas en inglés IEA, bajo los auspicios de la OCDE”. 


\section{Marco referencial}

(Gutiérrez, Pacheco Amigo, \& Rodríguez García, 2017) citando a Aula Santillana consideran que "Los hábitos de estudio son los modos constantes de actuación con que el escolar reacciona ante los nuevos contenidos, para conocerlos, comprenderlos y aplicarlos".

Para efectos del presente análisis se ha considerado como instrumento de recolección de datos el cuestionario propuesto por (Díaz Vega, 2006), el cual considera siete variables o factores; dicho cuestionario también utilizado en su proceso de investigación por los autores mencionados al inicio de este párrafo:

- Distribución de tiempo (DT), conlleva hacer distinción de actividades y su grado de importancia.

- Motivación hacia el estudio (ME), permite identificar el tipo motivación interna o externa en el estudiante.

- Distractores en el estudio (DE), estos pueden ser físicos, psicológicos, auditivos, visuales, incluso el tener un lugar propicio para estudiar.

Notas en clase (NC), identifica la habilidad para escuchar y tomar notas de forma sistemática y no de manera común.

- Optimización de lectura (OL), la capacidad de comprensión que se tiene para lograr la adquisición del conocimiento.

Preparación de exámenes (PE), permite que el estudiante efectué la planificación de los tiempos de estudio, evitando estudiar en el último momento.

Actitud hacia el estudio (AC) que consiste en poseer actitudes positivas frente al estudio y hacia los actores en el ámbito escolar.
(Villagrán, Cosgaya Barrera, \& Díaz Rosado, 2019) citando a Credee \& Kuneel (2008) consideran que las habilidades para el estudio se refieren al conocimiento de los alumnos sobre las estrategias y métodos de estudio apropiados y su habilidad para manejar el tiempo de estudio y otros recursos para lograr las demandas de sus tareas académicas, habilidades para organizar, resumir e integrar material. Otros autores como (Román, Olaguez Torres, \& Peña Peña, 2019) han identificado diversos factores que afectan el rendimiento académico de los estudiantes entre los que destacan aspectos personales, familiares, de índole actitudinal, así como no tener o conocer un método de estudio o técnica apropiada.

Como se aprecia en estos textos el entorno tanto interno como externo que rodea al estudiante también es decisivo en su proceso de aprendizaje y desempeño en su estudio.

Coincidimos con lo que expresan estos autores (Rivera, Valencia Gutiérrez, \& Vargas Contreras, 2017) que uno de los problemas que aquejan a los alumnos de licenciatura es el empleo de métodos y técnicas de estudio no apropiados, los cuales al repetirlos de forma constante durante el desempeño de sus tareas académicas, constituyen un mal hábito en su forma de enfrentar situaciones de aprendizaje. "El bajo rendimiento académico del estudiante refleja una situación que afecta a toda la comunidad estudiantil: estudiantes, padres, maestros y en consecuencia a toda la sociedad así lo afirman" (Lezama \& Alcántara Galdámez, 2017).

Efectivamente, nos pronunciamos a favor de que los pilares en el ámbito educativo son los alumnos, los directivos, los padres de familia y profesores, no obstante, debe tomarse en cuenta que el Estado y la Sociedad Civil de igual forma son entes importantes, ya que pueden verse afectados ante el fracaso escolar de sus estudiantes, generando problemáticas sociales graves, por mencionar algunas: la inseguridad y la infracción a las leyes. 
(Lezama \& Alcántara Galdámez, 2017) en su investigación retoman lo expresado por Jara et al. (2008, párr. 6) que dicen "las causas del bajo rendimiento de los estudiantes están relacionadas con la dificultad para el autoaprendizaje, escasos conocimientos básicos sobre las ciencias; así como la constante práctica de un aprendizaje memorístico", Por tal razón los autores del presente estudio se han interesado en conocer cuáles son los hábitos de estudio que sus estudiantes ponen en práctica, para posteriormente generar estrategias que se puedan implementar para apoyarles a mejorar su rendimiento académico y de esta forma se evite el rezago académico, la reprobación y la deserción. Además, es importante manifestar que se les puede ayudar por medio de objetos aprendizaje electrónico y así de esta manera aprovechar las Tecnologías de Información y Comunicación, que son herramientas aliadas al proceso de enseñanza- aprendizaje y en sí a la práctica docente.

Por otra parte, existen varias definiciones de objetos de aprendizaje para este artículo, nos basaremos en la expuesta por la IEEE (2002) que define a un objeto de aprendizaje como "cualquier entidad, digital o no digital, que puede ser usado para el aprendizaje, la educación o entrenamiento". Morales y Gutiérrez (2016) identifican que un OVA (Objeto Virtual de Aprendizaje o Virtual Learning Object) se refiere específicamente a los objetos de aprendizaje que corresponde a materiales digitales y que permite a los estudiantes aprender a su propio ritmo y de forma independiente. A este respecto, de estrategias a implementar consideramos la siguiente cuestión ¿Existen objetos de aprendizaje electrónicos que ayuden a mejorar los hábitos de estudio? La relación de los objetos de aprendizaje con los hábitos de estudio no se ha determinado de manera explícita y se han enfocado esfuerzos en la forma de hacer que los objetos de aprendizajes apoyen el aprendizaje significativo. Yahya y Yusoff (2011) en su investigación sobre un conjunto de metadatos de objetos de aprendizaje, resaltaron que los elementos esenciales del contexto y la reusabilidad permitirían a los usuarios ajustar la selección de objetos de aprendizaje de acuerdo a sus estilos de aprendizaje y su experiencia con otros objetos.
¿Qué características deben reunir los objetos de aprendizaje electrónicos que ayuden a mejorar los hábitos de estudio? De acuerdo con el proyecto "Aprendiendo con objetos de aprendizaje" APROA (2005) los objetos de aprendizaje virtuales deben tener las siguientes características: Ser auto-contenido, por si solo debe permitir lograr el objetivo para el cual fue diseñado. Sólo debe incorporar vínculos hacia documentos digitales que complementen el contenido. Ser interoperable, su estructura debe basarse en un lenguaje de programación XML, y contar con un estándar internacional de interoperabilidad (SCORM), que garantice su utilización en plataformas con distintos ambientes de programación. Ser reutilizable, puede ser utilizado por diversos educadores y estudiantes bajo distintos contextos de enseñanza y en diferentes tiempos. Ser durable y actualizable en el tiempo, es decir, que se encuentre respaldado por una estructura (Repositorio) que permita incorporar nuevos contenidos y/o modificaciones según las condiciones y los objetivos de aprendizaje. Ser secuenciable con otros objetos, es decir, que facilite la relación con otros objetos dentro de un mismo contexto de enseñanza. (p .5).

Hattie (2009) expone que hay tres categorías de habilidades de estudio: Las cognitivas que involucran la ejecución de una tarea o actividad, las meta-cognitivas que se relacionan con la autogestión, y las afectivas que se refirieren a la motivación y autoconcepto. En su trabajo sobre la enseñanza de habilidades de estudio, Fisher y Frey (2017) concluyeron que las habilidades de estudio son aprendidas si se incluyen actividades reflexivas, debe haber un entendimiento y compromisos en lo que se desea aprender y que las prácticas espaciadas en el tiempo son más efectivas. De esta manera se propone que un objeto de aprendizaje puede apoyar a los hábitos de estudio estableciendo algunas características adicionales como: a) Actividades de razonamiento sobre el objetivo de las actividades, b) un estimado del tiempo y esfuerzo requerido para usar el objeto de aprendizaje, y c) un ritmo de actividades paulatinas para poder ejecutar el objeto de aprendizaje o secuencia de objetos de aprendizaje. 


\section{Metodología}

La investigación que se propone tiene las siguientes características: es de tipo descriptiva, tiene un enfoque cuantitativo, con un diseño no experimental, meramente longitudinal (Sampiere, Collado Fernández, \& Baptista Lucio, 2014), derivado de que se presenta un análisis estadístico de los hábitos de estudio en tres periodos respectivamente, en una muestra de 17 estudiantes de Ingeniería en Sistemas Computacionales del Tecnológico Nacional de México, Campus Zacatecas Norte, es decir, se han recolectado datos del primero, segundo y cuarto semestre por medio de un cuestionario del autor (Díaz Vega, 2006), el cual considera siete variables o factores como son: Distribución de Tiempo (DT), Motivación hacia el Estudio (ME), Distractores en el Estudio (DE), Notas en Clase (NC), Optimización de Lectura (OL), Preparación de Exámenes (PE), y Actitud hacia el Estudio (AC).

Una vez obtenidos los datos se han capturado, se han utilizado técnicas estadísticas y se han interpretado para su análisis con el propósito de entender la situación con respecto de los hábitos de estudio que la estudiante práctica.

Para posteriormente, indagar sobre la existencia de objetos de aprendizaje electrónicos que ayuden a mejorar los hábitos de estudio.

\section{Resultados}

Es necesario considerar en este apartado la ponderación de los hábitos de estudio que se conforman de los puntos y porcentajes que se adquieren una vez contestado el instrumento de recolección de datos que propone el autor, (Díaz Vega, 2006), como se muestra en la Tabla 1. Cabe resaltar que una puntación mayor o igual a $21(70 \%)$, resulta un puntaje aceptable, sin embargo, se puede reforzar más por medio de la implementación de estrategias didácticas adecuadas.

\begin{tabular}{|r|r|}
\hline \multicolumn{2}{|c|}{ Ponderaciones } \\
Puntos & Porcentaje \% \\
\hline 30 & $100 \%$ \\
\hline 27 & $90 \%$ \\
\hline 24 & $80 \%$ \\
\hline 21 & $70 \%$ \\
\hline 18 & $60 \%$ \\
\hline 15 & $50 \%$ \\
\hline 12 & $40 \%$ \\
\hline 9 & $30 \%$ \\
\hline 6 & $20 \%$ \\
\hline 3 & $10 \%$ \\
\hline
\end{tabular}

Tabla 1 Ponderación en puntos y porcentajes Fuente: Elaboración propia, (2020)

En la Tabla 2, se puede apreciar la variación de los hábitos de estudio en base al promedio que se ha adquirido de forma grupal en los tres semestres:

\begin{tabular}{|c|c|c|c|}
\hline \multicolumn{4}{|c|}{ Promedio en porcentaje por semestre } \\
\hline HE & Primero & Segundo & Cuarto \\
\hline DT & 42 & 32 & 37 \\
\hline $\mathrm{ME}$ & 70 & 64 & 69 \\
\hline $\mathrm{DE}$ & 50 & 42 & 48 \\
\hline $\mathrm{NC}$ & 63 & 59 & 65 \\
\hline $\mathrm{OL}$ & 49 & 39 & 49 \\
\hline $\mathrm{PE}$ & 64 & 61 & 63 \\
\hline $\mathrm{AC}$ & 64 & 57 & 60 \\
\hline
\end{tabular}

Tabla 2 Promedio en porcentaje por semestre de los HE Fuente: Elaboración propia, (2020)

Los datos de la tabla anterior se muestran en el Gráfico 1, el cual se puede identificar un cambio significativo a la baja en los HE en segundo semestre en comparación con el primer y cuarto semestre, a excepción de $\mathrm{NC}$ que tiene un incremento y OL que se mantiene igual en primero y cuarto semestre:

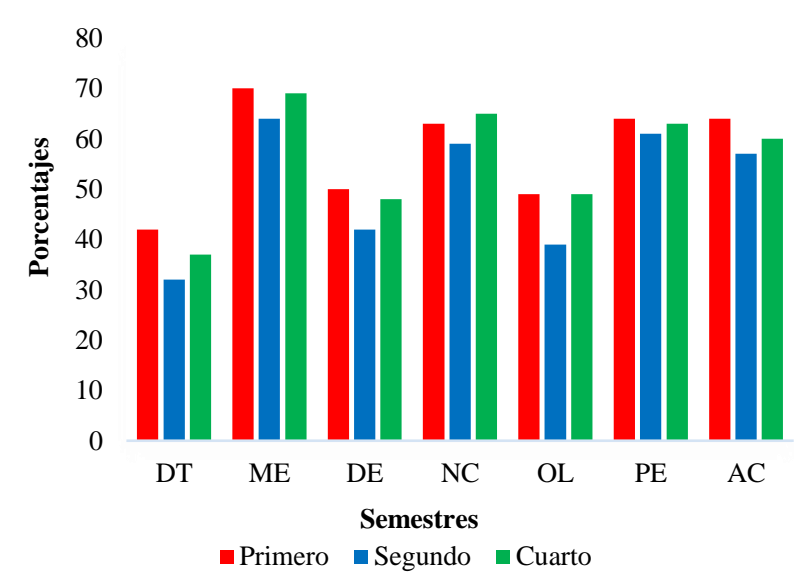

Gráfico 1 Promedio grupal de los HE en \%

Fuente: Elaboración propia, (2020) 
Otra técnica estadística que se ha utilizado para el tratamiento de los datos en el presente artículo es la desviación estándar, Tabla 3:

\begin{tabular}{|c|r|r|r|}
\hline \multicolumn{4}{c|}{ Desviación estándar } \\
\hline HE & Primero & Segundo & Cuarto \\
\hline DT & 12 & 10 & 12 \\
\hline ME & 10 & 10 & 11 \\
\hline DE & 10 & 15 & 11 \\
\hline NC & 11 & 14 & 12 \\
\hline OL & 14 & 16 & 12 \\
\hline PE & 8 & 13 & 13 \\
\hline AC & 9 & 9 & 9 \\
\hline
\end{tabular}

Tabla 3 Desviación estándar grupal de los HE Fuente: Elaboración propia, (2020)

En el Gráfico 2, se muestra el comportamiento de la desviación estándar de los hábitos de estudio de los 17 estudiantes de Ingeniería en Sistemas Computacionales del Tecnológico Nacional de México, Campus Zacatecas Norte, en la cual se puede ver una mayor variabilidad o dispersión en algunos datos lo que indica cierta heterogeneidad en algunos hábitos de estudio:

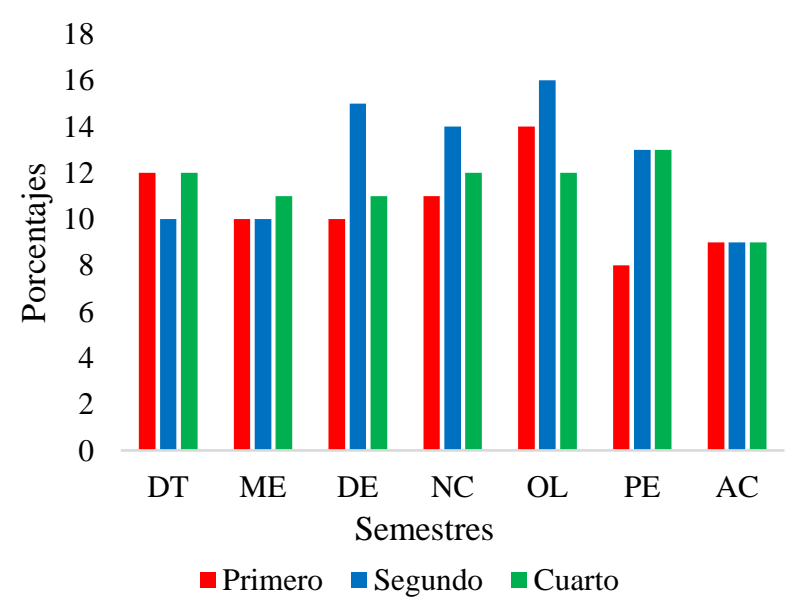

Gráfico 2 Porcentaje de la desviación estándar grupal de los HE por semestre

Fuente: Elaboración propia, (2020)

\section{Agradecimientos}

Nuestro agradecimiento al Tecnológico Nacional de México, Campus Zacatecas Norte (Instituto Tecnológico Superior Zacatecas Norte) por el apoyo proporcionado para llevar a cabo esta investigación.

\section{Conclusiones}

De acuerdo a los resultados del cuestionario de hábitos de estudio de Díaz Vega, aplicado a un grupo de alumnos de Ingeniería en Sistemas computacionales en su primer, segundo y cuarto semestre del Tecnológico Nacional de México, Campus Zacatecas Norte donde se encontró que la mayoría de ponderaciones de los hábitos de estudio denota que los alumnos no realizan estas actividades de manera efectiva y se puede observar que no hay mejoría significativa en el transcurso de los semestres, de igual forma la mayor variabilidad en la desviación estándar de las variables Distractores en el Estudio, Notas en Clase y Optimización de la Lectura indica que existe una heterogeneidad mayor entre los hábitos de los estudiantes, se concluye que es necesario establecer estrategias didácticas para que se les apoye a mejorar en estas prácticas. Para conseguir esto, se propone hacer uso de Objetos de Aprendizaje Electrónicos que cumplan con las siguientes características:

- Ser auto-contenido, por si solo debe permitir lograr el objetivo para el cual fue diseñado.

- Incorporar vínculos hacia documentos digitales que complementen el contenido.

- $\quad$ Su estructura debe de ser en XML.

- Contar con un estándar internacional de interoperabilidad (SCORM).

\section{Ser reutilizable.}

Se encuentre respaldado por una estructura (Repositorio) para incorporar nuevos contenidos y/o modificaciones.

- $\quad$ Ser secuenciable con otros objetos.

- Incluya actividades de razonamiento (reflexivas).

- Incluir actividades de lectura y comprensión.

- Incluir actividades que requieran tomar notas.

Se estime el tiempo y esfuerzo requerido para usar el objeto de aprendizaje. 
Exista un ritmo de actividades paulatinas.

Por lo anteriormente mencionado es de suma importancia que los docentes en su labor ordinaria implementen estrategias que apoyen a los estudiantes a mejorar los hábitos de estudio, más en estos tiempos donde el estudiante se enfrenta a un sinfín de distractores que les conlleva a desatender su responsabilidad académica y a presentar deficiencia en sus HE.

Por último, el ámbito educativo ha dado un giro significativo que lleva a los docentes y a los estudiantes a estar a la vanguardia con respecto del uso de las Tecnologías de la Información y Comunicación y que mejor impulsando el uso de Objetos de Aprendizaje Electrónicos que faciliten la responsabilidad del estudiantes en su práctica diaria en el proceso de aprendizaje, además, que se impulse en cada estudiante el dinamismo necesario para no entrar en la monotonía y aburrimiento; sino en el deseo de estar aprendiendo en todo momento, acción que se deriva de una práctica constante de HE.

\section{Referencias}

Acevedo, D., Torres, J. D., \& Tirado, D. F. (2015). Análisis de los Hábitos de Estudio y Motivación para el Aprendizaje a Distancia en Alumnos de Ingeniería de Sistemas de la Universidad de Cartagena (Colombia) Analysis of the Study Habits and Motivation for Learning of Distance Education Systems Enginee. 8(5), 59-66. https://doi.org/10.4067/S0718-

50062015000500007

Bajwa, N., Gujjar, A., Shaheen, G., y Ramzan, M. (2011). A comparative study of the study habits of the students from formal and nonformal systems of education in Pakistan. International Journal of Business \& Social Science, 2(14), consulted of E-Journal database. pp. 175-186, http://www.scielo.org.mx/scielo.php?script=sci nlinks\&ref=5159708\&pid=S0185$2760201200030000300004 \& \operatorname{lng}=\mathrm{es}$
Cartagena, M. (2008). Relación entre la autoeficacia, el rendimiento escolar y los hábitos de estudio de secundaria. Revista Iberoamericana sobre Calidad, Eficacia y Cambio en Educación, vol. 6 (3). http://www.rinace.net/arts/vol6num3/art3.pdf, consulta: abril 2015.

Castejón, J. (2014). Aprendizaje y Rendimiento Académico. Alicante, España: Club Universitario.

Chilca, M. L. (2017). Autoestima, hábitos de estudio y rendimiento académico en estudiantes universitarios. Propósitos y Representaciones, $5(1)$, 71-99. https://dialnet.unirioja.es/servlet/articulo?codig $\mathrm{o}=5904759$

Contreras, K., Caballero, C., Palacio, J., \& Pérez, A. M. (2008). Factores asociados al fracaso académico en estudiantes universitarios de Barranquilla (Colombia). Psicologia desde el Caribe. ISSN 0123-417X $\mathrm{N}^{\circ} 22$, juliodiciembre 2008.

Cruz Nuñez, F., \& Quiñones Urquijo, A. (1970). Hábitos de estudio y rendimiento académico en enfermería, Poza Rica, Veracruz México. Actualidades Investigativas En Educación, 11(3). https://doi.org/10.15517/aie.v11i3.10219

Díaz Vega, J. L. (2006). Aprende a estudiar con éxito (1a. ed.). Ciudad de México, México: Trillas.

Escalante, L., Escalante, Y., Linzaga, C., y Merlos, M. (2008). Comportamiento de los estudiantes en función a sus hábitos de estudio. Revista Electrónica Actualidades Investigativas en Educación, 8(2), 1-15, Instituto de Investigación en Educación, Universidad de Costa Rica, San José, Costa Rica.

Fisher, D., \& Frey, N. (2017). Teaching Study Skills. The Reading Teacher, 71 (3), 373- 378. https://doi.org/10.1002/trtr.1625

Gutiérrez, J. L., Pacheco Amigo, B. M., \& Rodríguez García, F. J. (2017). Formación de hábitos de estudio: Comparación entre alumnos de secundaria y alumnos universitarios. Revista de Docencia e Investigación Educativa, 3(8), 55-63. 
Hattie, J. (2009). Visible learning: A synthesis of over 800 meta- analyses relating to achievement. New York, NY: Routledge.

Hernández, C., Rodríguez, N. y Vargas, A. (2012). Los hábitos de estudio y motivación para el aprendizaje de los alumnos en tres carreras de ingeniería en un tecnológico federal de la ciudad de México. Revista de la educación superior, 41(163), 67-87. Recuperado de http://www.scielo.org.mx/scielo.php?script=sci _arttext\&pid=S018527602012000300003\&lng $=$ es\&tlng=es

IEEE Standard for Learning Object Metadata," in IEEE Std 1484.12.1-2002, vol., no., pp.1-40, 6 Sept. 2002

Lezama, O. B., \& Alcántara Galdámez, N. J. (2017). Hábitos de estudio y rendimiento académico en estudiantes universitarios. Innovare, 6(2), 19-34.

Morales Martín, L. Y., Gutiérrez Mendoza, L., \& Ariza Nieves, L. M. (2016). Guía para el diseño de objetos virtuales de aprendizaje (OVA). Aplicación al proceso enseñanzaaprendizaje del área bajo la curva de cálculo integral. Revista Científica General José María Córdova, $14(18), \quad 127$. https://doi.org/10.21830/19006586.46

Perrenoud, P. (1996). La construcción del éxito $y$ del fracaso escolar. $2^{a}$ ed., Madrid. ed. Morata.

Proyecto educacional de Chile: "Aprendiendo con objetos de aprendizaje" (APROA). (2005). Manual de buenas prácticas para el diseño de objetos de aprendi- zaje. Recuperado el 15 de abril de 2020 de: http:// www.aproa.cl/

REAL ACADEMIA ESPAÑOLA: Diccionario de la lengua española, 23. a ed., [versión 23.3 en línea]. <https://dle.rae.es> [02 de abril 2020].

Rivera, D. L., Valencia Gutiérrez, M. d., \& Vargas Contreras, J. A. (2017). La habilidad de autoevaluación de hábitos de estudio. Revista Varela, 18(49), 69-81.

Román, P. E., Olaguez Torres, E., \& Peña Peña, E. (2019). Hábitos de estudio en estudiantes de ingeniería mecatrónica: base para incidir en el índice de aprobación. Opuntia Brava, 317-332.
Sampiere, R. H., Collado Fernández, C. C., \& Baptista Lucio, P. (2014). Metodología de la Investigación. México: McGraw-Hill.

Sánchez, C. S. (2002). Diccionario de las Ciencias de la Educación. 18va ed. México. ed. Aula Santillana.

Torres, M., Tolosa, I., Urrea, M., Monsalve, A. (2009). Hábitos de estudio vs fracaso académico. En Revista Educación de la Universidad de Costa Rica, Vol. 33, Núm. 2. http://redalyc.uaemex.mx/pdf/440/4401205800 2.pdf, consulta: enero de 2012.

Villagrán, A. C., Cosgaya Barrera, B. R., \& Díaz Rosado, M. (2019). Hábitos de estudio presentes en estudiantes de nivel superior. AMMCI, Memorias de Congresos, 2(4), 406417.

Villegas, C., Muñoz, F., \& Villegas, R. (11 de Septiembre de 2016). Hábitos de estudio de los alumnos en el área de Química Orgánica. Obtenido de Revista Biotecnia, Vol.XI,no3 Sept-Dic 2009: http://biotecnia.ojs.escire.net/index.php/biotecni a/article/viewFile/72/66

Yahya, Y., \& Yusoff, M. (2008). Towards a comprehensive learning object metadata: Incorporation of context to stipulate meaningful learning and enhance learning object reusability. Interdisciplinary Journal of ELearning and Learning Objects, 4(1), 13-48. 